\title{
A Novel Hierarchical Coding Scheme Based on Polar Mass Distribution
}

\author{
Levent Öktem and Levent Onural \\ Electrical and Electronics Engineering Department \\ Bilkent University, 06533 Bilkent, Ankara, TURKEY \\ phone: $+90-312-266-4307$
}

\begin{abstract}
A novel method for coding arbitrarily shaped binary objects is presented. The main idea is expressing the binary object in terms of radial closed curves, that is, closed curves for which radius versus angle is a function. These functions and the simple rules for combining them uniquely represent the object. The data compaction property of the method and the flexibility of the introduced loss are shown. Experimental results are presented, with a comparison to G3 fax standard. Possible application areas are discussed.
\end{abstract}

\section{INTRODUCTION}

Boundary coding has gained more importance in the last years, with the increasing popularity of segmentationbased image coding methods. There are a huge amount of boundary coding techniques. The most popular are chain codes [1], fitting line segments, B-spline representation , autoregressive models ${ }^{[2]}$ and morphological methods [3]

Chain codes are the indices for the difference vector of two neigboring pixel coordinates on a contour. Some of the redundancy in the chain codes is eliminated by statistical methods, such as arithmetic coding or runlength coding. Chain codes are lossless codes. On the other hand, some loss must be introduced for high compression. So, prior to coding the boundary is forced to certain shapes, and not all the chain combinations are allowed. This serves as a loss to increase compression.

One of the most convenient boundary representations is B-spline matching. B-splines are a set of piecewise polynomial functions, used for approximating a contour in an interval. For matching B-splines, first the contour is separated into intervals by control points. These points are generally the ones that have high curvature. Then, B-splines are fit to these intervals. If the result is not satisfactory, the number and locations of the control points are revised, and B-spline fitting is repeated. This iterative procedure is repeated until the resulting approximation is found satisfactory. The control points and B-spline type indices represent the curve.

One other popular region boundary coding method is morphological shape representation. In this method, the region is considered as a binary object. The binary object is decomposed into simple geometric objects and a rule (a morphological operation) of how to synthesize them to get the object. The simple geometric objects and the rules are transmitted.

Morphological methods are being more popular than the others, and there are three main reasons for that:

- The representation parameters of morphological methods are convenient for the analysis of the shape i.e. they are intelligent representations.

- These representation parameters can be coded efficiently.

- The shape can be represented at various detail levels.

There are many methods having either of the above properties, but having all is a virtue. Section 2 describes a boundary coder which has all of the above properties, while not being a morphological method in the conventional sense.

\section{THE METHOD}

The approach of design is modifying an intelligent boundary representation scheme to be efficiently codable and to have multiresolution property.

Fourier descriptors ${ }^{[4]}$ have been used for shape analysis of closed curves. They are useful in analysis since rotation, scaling and translation of objects can be represented with one parameter. They are not, however, popular in coding. This is because rectangular Fourier descriptors do not have an energy compaction property for common binary objects, and polar Fourier descriptors are restricted to radial curves only.

0-7803-1772-6/94/\$3.00 (C) 1994 IEEE 
Polar Fourier descriptors can be modified to be applicable to any binary object, as follows.

\section{Definitions:}

- Object: Any connected set of full pixels.

- Radial object: An object for which there exists a point in the set such that any ray originating from that point intersects the contour at only one point.

- Polar hull of the object $A(P H(A))$ : The minumum radial object that covers $A$.

- Residue of the object $A(R(A))$ : The set of points contained in $P H(A)$, but not contained in $A$.

- Polar mass distribution of an object $A$ at the angle $\theta\left(P M D_{A}(\theta)\right)$ : The total distance that the ray originating from the center of mass of $A$ with angle $\theta$ travels inside the object.

Observation: $R(A)$ and $P M D_{A}(\theta)$ uniquely represent $A$.

Coding Scheme:

For coding an object $A$, its center of mass and the transform code (e.g. DCT ${ }^{[5]}$ ) of $P M D_{A}($.$) are Huff-$ man ${ }^{[6]}$ coded. $R(A)$ is regarded as a set of objects, and each object is coded the same way as $A$. In the end of this recursion, a tree representation of the object is obtained.

Properties:

The coding scheme can represent any binary object. Some transforms (e.g. DCT) of PMD are observed to have good data compaction for several common images. So, it may be used for data compression.

A controlled loss can be introduced to the tree representation of the object, i.e some parts of the tree can be omitted. Furthermore, the transform code coefficients of PMD can be quantized. This allows the representation of the object at various details.

2-D motion and scaling of the object can be analized and represented easily via the tree representation. So, it can be used for motion compensation.

It can be extended to grey-scale images. For greyscale images, $P M D(\theta)$ is replaced by $P M D(\theta, \phi)$.

\section{RESULTS}

With experiments on several binary objects (e.g. silhouettes of a hand, a head-and-shoulder, and printed text), it has been observed that

- The trees do not go deep.

- For the DCT of PMD's, most of the energy is cumulated in the first few coefficients.

Due to this, high compression ratios can be achieved with very little degradation. Figure 1 shows the original and coded text. The compression ratio is $25: 1$. This is three times the compression ratio of G3 fax standard for coding these images.

\section{0 perform a $r$ nt density on gous electrica $r$, which is $t r$ communication}

(a)

\section{to perform a $r$ ni density on gous electrica $r$, which is $t r$ communication}

(b)

Fig 1. (a) Original text (b) coded text.

\section{CONCLUSIONS AND FUTURE WORK}

A novel hierarchical coding scheme for coding binary objects has been presented. The advantage of the system is having all three of the properties listed in section 1 : it is an intelligent representation whose parameters can be coded efficiently and hierarchically. This hierarchy brings the multiresolution property.

The presented experiment results do not say much about the intelligence of the system. Future work will be focused on exploiting the properties of the representation that will be useful in motion and shape analysis. After this stage is completed, the presented coder will be an essential part of a segmentation based image sequence coder. 


\section{REFERENCES}

[1] T. Kaneko, and M. Okudaira, "Encoding of Arbitrary Curves Based on the Chain Code Representation", IEEE Transactions on Communications, vol.s9, no. 7, pp.697-707, July 1985.

[2] A. K. Jain, Fundamentals of Digital Image Processing. Prentice Hall International Editions, 1989.

[3] The RACE MORPHECO Group, "Morphological Segmentation-Based Coding of Image Sequences", COST 211 ter European Workshop on New Techniques for Coding of Video Signals at Very Low Bitrates, Hannover, December 1-2, 1999.

[4] E. Persoon, and K.-S. Fu, "Shape Discrimination Using Fourier Descriptors", IEEE Transactions on Systems, Man, and Cybernetics, vol.7, no.3, March 1977.

[5] K. R. Rao. ve P. Yip, Discrete Cosine Transform: Algorithms, Advantages, Applications. Academic Press, Inc, 1990.

[6] D. A. Huffman, "A Method for the Construction of Minimum Redundancy codes", Proc. of ERE, $p p$. 1098-1101, September 1952. 